\title{
Treatment of Coronary Bifurcations: Simplify, but not excessively
}

\author{
Áurea J. Chaves
}

$\mathrm{T}$ he percutaneous treatment of coronary bifurcation lesions is, together with the treatment of lesions in degenerate saphenous vein grafts and chronic occlusions, one of the biggest challenges that an interventional cardiologist faces in daily practice. Several techniques for treating bifurcations have been described in addition to algorithms to indicate these techniques based on the calibre of the secondary branch and its angulation on the level of lesion involvement, but so far none has surpassed the simplicity of the provisional technique. In this issue of the Revista Brasileira de Cardiologia Invasiva (RBCI), in an excellent editorial, Buysschaert and Verheye from the Antwerp Cardiovascular Institute, ZNA Middelheim (Antwerp, Belgium), explain the current consensus on the treatment of bifurcations and go beyond this, when they discuss whether post-dilation with kissing-balloon in the provisional technique should be performed and whether systematic pre-dilation of the side branch, particularly in complex bifurcation lesions, is recommended. They discuss the article by Costa et al. of the Instituto Dante Pazzanese de Cardiologia (São Paulo, SP, Brazil), which evaluated the impact of side branch pre-dilation in true bifurcation lesions, with side branch lesions extending $5 \mathrm{~mm}$ beyond the ostium. They conclude the discussion by recommending a scenario in which they believe side branch pre-dilation should be indicated.

In another excellent editorial, Nakagawa and Ikeno from the Falk Cardiovascular Research Center, School of Medicine, Stanford University (Stanford, CA, USA), introduce us to the world of drug-eluting balloons. They comment on why the drug-eluting balloons approved for clinical use in Europe only use the drug paclitaxel as a neointimal proliferation inhibitor and describe the new sirolimus-eluting balloon, which is still in the experimental phase. They explain the importance of bench testing to detect the capacity of the released drug to infiltrate the arterial wall, to quantify the drug, and to verify whether it remains in the deeper layers of the vessel. They show the key role of the excipient as a method of antiproliferative drug delivery by the balloon and the need to prove the effectiveness of balloons in preclinical studies. In this regard, they discuss the original work by Takimura et al. from the Instituto do Coração do Hospital das Clínicas da Faculdade de
Medicina da Universidade de São Paulo (São Paulo, SP, Brazil), who observed different neointimal responses, as assessed by optical coherence tomography and histopathology, of various excipient:drug formulations of the sirolimus-eluting balloon in an animal model. They complement their recommendations by noting that additional long-term data and pharmacokinetics evaluation are some of the steps that must be taken before performing clinical trials.

Other original studies were also published in this issue of RBCI. Zago et al., from the Hospital de Clínicas de Porto Alegre (Porto Alegre, RS, Brazil), identified genes involved in smooth muscle cell structural and functional protein synthesis associated with neointimal hyperplasia in human atheromatous plaques obtained by guided atherectomy after coronary stent implantation. The finding that the atheromatous plaque has a significantly inherent predisposition to neointimal formation before stenting could pave the way for the development of new antiproliferative drugs in the search for a definitive solution to coronary restenosis. Abreu-Silva et al., from the Cardiovascular Research Center (São Paulo, SP, Brazil), present results of the PAX-B study, an international multicentre study that evaluated a non-polymeric paclitaxel-eluting stent in a complex angiographic scenario, with angiographic follow-up at nine months and clinical follow-up after 12 months. In an interesting discussion, they debate the problems caused by polymers, but recognise their advantage in regulating the release kinetics of antiproliferative drugs, which are crucial for neointimal hyperplasia suppression, as well as tested alternatives for non-polymeric stents, such as the structural modification of the abluminal surface of metal struts.

Other articles complement this issue of $\mathrm{RBCl}$ with topics of great interest, such as the adjunctive use of fondaparinux in percutaneous coronary intervention in patients with acute coronary syndrome, the ten-year prognosis of young patients with coronary artery disease treated percutaneously, the current results of percutaneous coronary intervention in diabetic patients, the association between oral health and lesions detected on coronary angiography, the approach to non-aortic ostial coronary lesions using the Szabo technique, 
the treatment of complicated type B aortic dissection, and the initial experience of PDA closure using the Amplatzer ${ }^{\circledR}$ Vascular Plug II.

Finally, we would like to communicate that the logo of the Brazilian Society of Interventional Cardiology has been updated and will, from this issue onward, replace the previous logo on the cover and in institutional communications published in $\mathbf{R B C I}$.

Enjoy your reading!

Áurea J. Chaves

Editor 